\title{
El acercamiento al contexto profesional como móvil para indagar sobre las TIC: un estudio cualitativo ${ }^{1}$
}

\section{The approach to the professional context as a motive to investigate about ICT: a qualitative study}

\author{
María Carmen RICOY LORENZO, Maria João V. S. COUTO \\ Universidad de Vigo
}

Recibido: Mayo 2012

Aceptado: Junio 2012

\section{Resumen}

Con este trabajo se trata de conocer la percepción que tiene un grupo de estudiantes del máster de formación del profesorado de secundaria sobre los beneficios y controversias que genera la inclusión de las TIC en los centros, a partir de su inmersión en las prácticas externas. La investigación se abordó a través de un estudio cualitativo, utilizando la técnica de la entrevista. Se han implicado un total de 20 participantes que cursaban el máster en la Universidad de Vigo. Los resultados revelan que el uso de las TIC mejora la motivación, incrementa la autonomía, la predisposición por compartir experiencias en el alumnado, y suscita un mayor nivel de compromiso en el profesorado. Se descubren obstáculos relacionados con la deficiente formación docente y con el mal uso de las TIC.

Palabras clave: TIC, formación inicial, universidad, prácticas externas, desarrollo profesional, educación secundaria.

\begin{abstract}
With this work is intended to know the perception of a students' group who attended the master teacher training for secondary education about the benefits and controversies generated by the inclusion of ICT in schools, from their immersion in external practices. The investigation was approached through a qualitative study, using an interview technique. There were involved a total of 20 participants who attended the master at University of Vigo. The results reveal that ICT use improves the motivation, increases the autonomy, the predisposition to share experiences in the pupils, and provokes a greater level of teacher commitment. It turns out obstacles related to poor teacher training and misuse of ICT.
\end{abstract}

Keywords: ICT, initial training, university, external practices, professional development, secondary education.

\footnotetext{
${ }^{1}$ Este trabajo está vinculado a un proyecto de investigación (ref.: PGIDIT06PXIB374371PR) financiado por la Junta de Galicia. De modo que aprovechamos para manifestar nuestro agradecimiento a la entidad subvencionadora, así como a los participantes.
} 
Las Tecnologías de la Información y Comunicación (TIC) son en la actualidad, en mayor o menor medida, un recurso al alcance de los docentes en todos los niveles educativos, y los principales impulsores de su inclusión han de ser los propios profesores. Además hemos de tener en cuenta que estos dispositivos digitales se caracterizan fundamentalmente por su potencial para contribuir a la modernización e innovación educativa. No en vano distintos cambios que afectan al contexto educativo están apoyados y/o derivan del uso de las TIC. Por ejemplo, para Murga y García (2010) el blog es simultáneamente un instrumento metodológico innovador y una herramienta informática adecuada para motivar al alumnado en el proceso de formación.

La aplicación apropiada de los recursos digitales depende, entre otros aspectos, del consonante pedagógico. Por ello, la incorporación de metodologías acordes con la implantación de las TIC supone avanzar en una buena dirección, cuando de entrada éstas cuentan con mecanismos para originar transformaciones relevantes, al menos, a medio plazo. Con todo, es de reconocer que no siempre el uso de las TIC ha desencadenado buenas prácticas, dado que como ha afirmado algún autor también se reproducen viejas estrategias con la introducción de nuevos medios didácticos (Cabero, 1994; Area, 2010). Para Flichy (1995) las tecnologías no garantizan las prácticas innovadoras, a no ser que exista un cambio real. Sin embargo sus posibilidades se convierten en hechos categóricos, aunque el desarrollo de las actividades educativas también dependa del tipo de entorno al que vayan a destinarse (Jamieson, Fisher, Gilding, Taylor y Trevitt, 2000).

La utilización de las TIC nos tiene que llevar a reflexionar sobre sus aplicaciones para tratar de promover la renovación. De hecho Chen, Yu y Chang (2007) sostienen que la integración curricular de las herramientas digitales constituye un nuevo paradigma formativo y que estos recursos son imprescindibles para la innovación. Por ello, es recomendable que la renovación pedagógica se impulse a través del uso de las TIC y que se enmarque dentro de un contexto de cambio global.

A pesar de la diversidad de problemáticas imperantes sostenemos que las TIC facilitan la innovación en los centros educativos. Por ello, formarse para su aplicación se convierte en una necesidad personal y colectiva en la sociedad actual. No podemos obviar que descubrir el potencial y las posibilidades de los recursos digitales es una tarea a desarrollar en la formación inicial y continua, tanto de modo personalizado como grupal. Sin olvidar que es una obligación de la administración la alfabetización digital, así como una exigencia y un derecho en la formación de los futuros docentes, y hasta donde fuese preciso también en la continua.

El trabajo que nos ocupa se focaliza hacia el análisis de los puntos fuertes y las controversias que percibe el alumnado del máster de formación del profesorado de secundaria sobre el uso de las TIC, después de la realización de sus prácticas externas (prácticum) en los centros educativos. Siguiendo a Sierra y Pérez Ferra (2007) la reflexión sobre la acción que se produce a posteriori puede orientar oportunamente la práctica profesional en el futuro. De hecho como apunta Cardona (2008) las 
situaciones de la práctica real requieren del cultivo de la reflexión para encontrar soluciones adecuadas a los problemas emergentes.

El análisis sobre la identidad docente en la formación inicial del profesorado de secundaria entraña gran importancia, en la medida en que se indague sobre sus conocimientos y creencias previos con el objeto de que reflexionen y para ayudarles a interpretar sus experiencias pre-profesionales (Marcelo, 2009). También se sabe que el uso de las TIC por parte del profesorado depende, entre otros elementos, de las creencias que éste ostenta en torno a las mismas, así como de la concepción sobre la enseñanza y del propio estilo docente (Ertmer, 2005), además de la autoconfianza necesaria para su aplicación en el aula (Almerich, Suárez, Belloch y Orellana, 2010).

Azcárate y Cuesta (2005) entienden que el profesorado es capaz de originar cambios consistentes y de impulsar su evolución profesional. Para ello, ha de ser consciente de sus acciones a partir de la reflexión sobre sus experiencias y de la comprensión de la realidad. Una vez que, la profesión docente requiere de un proceso integrado por distintas fases y ámbitos interrelacionados, teniendo la experiencia de la formación inicial mucha importancia en los aprendizajes del futuro profesorado (Sandoval, 2009). Reflexionar sobre la acción posteriormente constituye un pilar esencial para analizar y entender el proceso de aprendizaje durante el periodo de formación. Además, siguiendo a Fernández March (2008), la reflexión sobre la práctica es condición indispensable para garantizar que el docente pueda adaptarse y prever los cambios que la nueva sociedad del conocimiento exige. De aquí la importancia de promover en los futuros profesores la capacidad crítica, de apertura y toma de conciencia ante las constantes transformaciones que se producen de forma irremediable.

La complejidad que rodea a la práctica educativa hace necesario que el profesorado esté atento a las contingencias del contexto, que cuestione las situaciones problemáticas y trate de buscar nuevas alternativas para afrontarlas (Latorre, 2007). Por ello, es particularmente importante para mejorar la formación inicial del alumnado saber como piensa para orientarlo y fomentar su juicio crítico. No olvidemos que la participación, la reflexividad y la toma de conciencia son componentes cruciales para su desarrollo profesional. Es más, como indica García Ruiz (2012) la universidad del siglo XXI no debe abdicar del que ha sido uno de sus principales roles a lo largo de la historia: el de enseñar a los estudiantes a pensar críticamente. Además, como institución de formación superior tiene entre sus fines la preparación de profesionales cualificados para desempeñar múltiples responsabilidades en distintos campos laborales (López Ruiz, 2011).

Como a Porlán, Martín, Rivero, Harres, Azcárate y Pizzato (2010) nos preocupan los obstáculos que encuentran el alumnado y los docentes para el cambio, así como para construir su conocimiento profesional. De modo que consideramos la necesidad de poner énfasis en investigar sus percepciones, transiciones y progresos cuando participan en procesos de formación. Orientar a los estudiantes en su periodo de formación a partir de la práctica, a través de la reflexión, contribuye a la toma de 
conciencia de la realidad, así como a desarrollar competencias y actitudes que les permitirán en el futuro continuar aprendiendo de la experiencia.

Los estudios a partir de las actividades prácticas también son interesantes para poder propiciar y ampliar en los docentes de universidad una base de conocimientos sobre las concepciones de los estudiantes que se relacionan con el contexto profesional (De Oliveira y De La Rocque, 2011). Con otras investigaciones (Little, Gearhart, Curry y Kafka, 2003), que se han llevado a cabo desde distintas disciplinas, se descubre que produciendo situaciones de reflexión en el alumnado y analizándolas se amplían las oportunidades de auformación del profesorado de la educación superior, así como las de compartirlas con otros colegas y la comunidad científica. Además el profesorado (y consideramos que también el alumnado) se siente reforzado con la presencia y el análisis sobre el uso de las TIC, encaminando este último hacia la resolución de problemas prácticos: introducción de recursos e innovaciones metodológicas, búsqueda de estrategias eficaces de aprendizaje y apoyo, comunicación entre profesorado y alumnado, etc. (Fombona y Pascual, 2011).

\section{Objetivos de investigación}

La finalidad central, del estudio aquí presentado, está dirigida a indagar sobre los pros y los contras que encuentran en el uso de las TIC los estudiantes del máster de formación del profesorado de secundaria, a partir de su inmersión en el contexto profesional, una vez realizadas las prácticas externas.

Como objetivos específicos se han definido los siguientes:

- Determinar los beneficios que consideran que genera el empleo de las TIC en el proceso de enseñanza-aprendizaje.

- Descubrir las controversias que asocian a la inclusión de las TIC en el contexto educativo.

- Delimitar los cambios o mejoras que le atribuyen a la utilización de las TIC en los centros de secundaria.

\section{Método}

\section{Contexto}

Aunque no es objeto de este trabajo abundar en su primera parte dedicada al desarrollo de la entrevista como estrategia didáctica con los estudiantes, ofrecemos una breve descripción del contexto en el que se sitúa para facilitar el sentido del encuadre general.

Teniendo en cuenta el tiempo de dedicación que requiere, hemos planteado la entrevista como una actividad voluntaria al alumnado del máster del profesorado de secundaria, en consecuencia esta estrategia de formación tuvo carácter limitado. Su realización quedó supeditada a la decisión de aquellos estudiantes que de forma 
discrecional eligen ser entrevistados, y a la solidez e interés que presentaba la información facilitada.

A partir del uso de la entrevista como estrategia didáctica, en la materia de "Innovación e Investigación" del máster de formación del profesorado de secundaria, en un primer momento tratamos de favorecer el desarrollo de la competencia reflexiva del alumnado (que es de vital importancia en la formación inicial). Dialogamos y reflexionamos con ellos con la pretensión de potenciar prácticas innovadoras en el marco de la adaptación de la docencia al Espacio Europeo de Educación Superior.

Desde nuestro estilo docente intentamos formar a los estudiantes utilizando estrategias de aprendizaje activo y que promueven juicios argumentativos, asimismo indagamos sobre nuestra propia práctica de forma metódica (Flick, 2004). Un hecho importante, como resaltan Cabero y Martín (2011), es que la actitud de los estudiantes condiciona las percepciones sobre las dinámicas empleadas por el profesorado, de ahí la importancia de incluir medidas para autoincentivarnos también los docentes.

Somos conscientes, como indica Perrenoud (2007), de que la formación inicial tiene que preparar a los futuros docentes para observar y reflexionar durante y después de la acción. Con todo, asumimos que no siempre las estrategias centradas en el alumnado provocan aprendizaje sistemático, pero contribuyen al conocimiento de la realidad y a la interiorización de los contenidos. Este autor incide, además, en que la competencia reflexiva está en el centro de todas las de tipo profesional constituyendo parte de su desarrollo, así como para favorecer la articulación teórico-práctica y la generación de nuevas actitudes.

Conocedoras del doble interés que deriva de la recogida de información con la técnica de la entrevista como estrategia formativa y de investigación, como adelantamos, en este estudio no fue generada en exclusividad para la primera, ya que con buen criterio tratamos también de utilizar los datos de campo obtenidos (Suárez Pazos y Membiela, 2011). De modo que pretendemos indagar mediante un análisis en profundidad sobre las opiniones, comentarios e impresiones del grupo seleccionado, con el objeto de adentrarnos en la percepción que tienen del uso de las TIC, a partir de su inmersión en los centros de secundaria.

La presente contribución, a su vez, está vinculada con una investigación más amplia desarrollada a través de una metodología mixta cuyo proyecto hemos referenciado inicialmente. El trabajo aquí recogido se ha abordado a partir de un estudio de caso, de carácter cualitativo. Dado que para Stake (2007) el estudio de caso puede ser de una sola persona o de un grupo, el emprendido es de tipo instrumental y permite analizar el punto de vista, las opiniones e impresiones desde la perspectiva del alumnado que formaba parte de dos aulas (subcasos). Además, siguiendo a Coller (2005) esta metodología posibilita la descripción, comprensión y conocimiento de la situación objeto de análisis, dentro del contexto elegido.

Partimos del hecho de que cualquier metodología de investigación es válida si se aborda con la sistematización y rigor adecuado, y tanto desde un planteamiento cuantitativo como cualitativo o mixto (Yin, 2006). En este trabajo aportamos la parte 
del estudio que se ha realizado desde el enfoque cualitativo, promovido a partir del proyecto referido e inmerso en la dinámica pedagógica descripta.

Sostenemos al igual que González Cubillán (2009) que los estudios cualitativos posibilitan el conocimiento de la situación analizada desde su propia singularidad. Por ello, hemos analizando las narrativas procedentes de las entrevistas para profundizar en su contenido y brindar, desde este trabajo, a otros profesionales y a la comunidad científica los resultados y conclusiones obtenidos. La finalidad de la metodología utilizada no es la generalización de los resultados o conclusiones sino profundizar sobre un caso concreto y contribuir a la comprensión de la realidad (Yin, 2006). Con todo, las presentadas pueden ser susceptibles de extrapolarse, ya que el estudio de casos aporta tópicos sustantivos de generalización analítica a otros contextos (Duarte, 2008).

\section{Participantes}

La investigación cualitativa no se basa en un muestreo estadístico representativo, ni es factible ni necesaria una muestra muy amplia de participantes (Stake, 2007). Partiendo de lo anterior la selección de los participantes estuvo relacionada con la accesibilidad que se tenía a los mismos y la disponibilidad que mostraban. Realizamos su elección a través de criterios que permiten recoger la máxima información objeto de estudio desde las circunstancias en las que nos encontrábamos, y a partir de las condiciones más propicias (Vale, 2000). De modo que, siguiendo a McMillanm y Shumacher (2005), para la selección se han considerado las posibilidades de accesibilidad que se tenía a las aulas y al alumnado, así como la aceptación voluntaria por parte del mismo para ser entrevistado.

Los participantes que se han implicado en este estudio son algunos de los titulados universitarios que cursaban el máster de formación del profesorado de secundaria de la Universidad de Vigo, en el curso académico 2009-2010. En total se han involucrado voluntariamente 20 titulados del Campus de Ourense. De las dos especialidades, la de Ciencias Experimentales (conformada por 3 itinerarios) y la de Ciencias Sociales (cuenta con 2 itinerarios) impartidas en el Campus de Ourense, se logró disponer para la recogida de información y reflexión de alrededor de la mitad de los estudiantes de los itinerarios de: "Tecnología e Informática" (10 participantes) y "Geografía e Historia" (10 participantes). En esta selección se ha cuidado la equidad entre sexos por itinerario, implicándose cinco varones y otras tantas mujeres de cada uno. La edad de este alumnado estaba comprendida entre los 23 y 35 años, con una media de 29 años. En términos generales se trata de jóvenes titulados.

\section{Instrumento y análisis de la información}

Como indicamos se ha utilizado para la recogida de información la técnica de la entrevista cuyo guión fue elaborado ad hoc materializándose en siete preguntas. Estas cuestiones fueron diseñadas a partir de los elementos objeto de reflexión con el alumnado que, además, hemos sometido a análisis. El protocolo utilizado en esta 
entrevista es de tipo semi-estructurado y está precedido de los datos de identificación de los participantes, que hemos rescatado para el presente trabajo junto con tres interrogantes centrales de la misma:

- ¿Qué ventajas consideras que tiene la inclusión de las TIC en el proceso de enseñanza-aprendizaje en los centros de secundaria?

- ¿Qué Obstáculos le atribuyes al uso de las TIC con el alumnado de educación secundaria?

- ¿Cuáles consideras que son los cambios o mejoras que originan las TIC en secundaria?

El guión a través de la unificación de sus preguntas nos permitió obtener información contextualizada y fue de gran utilidad para sistematizarla, así como para proceder al análisis de su contenido. Las diferentes entrevistas fueron realizadas, previa confirmación, por el profesorado de la materia de "Innovación e Investigación" del máster de secundaria, en el momento y lugar acordado con cada entrevistado, de forma individual. La duración de las conversaciones ha oscilado entre 40 y 50 minutos. El contenido en su totalidad se ha grabado en formato audio (de forma anónima) para su posterior transcripción, contando con la autorización de los implicados.

Los textos producidos, a partir de las respuestas de los participantes, fueron examinados en su integridad mediante un análisis de contenido. Su categorización inicial (primaria o principal) se determinó a través del enunciado de cada pregunta planteada en la entrevista, y se representa sintéticamente como referente de cada subepígrafe del apartado de resultados. Emprendimos el análisis con estas categorías principales por considerarlas directrices. Las subcategorías (de $2^{\circ}$ y $3^{\circ}$ nivel, según los casos) derivaron de la información obtenida desde una concepción naturalista. Este sistema de codificación se configuró a partir de la realización de las transcripciones en bruto de los textos grabados, identificando núcleos de ideas con sentido completo (Gürtler y Huber, 2007).

El análisis de contenido se desarrolló por medio del programa de Analysis of Qualitative Data (AQUAD), en su versión seis para texto. Una vez terminado el proceso de codificación se importó, a través del programa Excel 2007, el recuento de frecuencias (se denotarán en las tablas sucesivas con la inicial/símbolo $f$ ) de las respectivas subcategorías para revelar la cantidad de manifestaciones de los participantes. La identificación de la frecuencia permite conocer el nivel de preponderancia y singularidad de las subcategorías, aunque con ello no tratamos de enfatizar la faceta cuantitativa. Asimismo, a través de los análisis abordados se adquieren resultados globales y parciales de cada participante, y del conjunto de los implicados.

La cantidad de entrevistados no se concretó inicialmente, sino que se estableció a partir de la disponibilidad comunicada por el alumnado para participar, y del nivel de saturación de los datos obtenidos. Una vez comprobado que un número mayor ya no afectaba a los resultados interrumpimos definitivamente el proceso de codificación (Miles y Huberman, 1984). 
El análisis de contenido ha sido realizado por pares de investigadoras, concretamente por una profesora responsable de la docencia en la materia de "Innovación e Investigación" del itinerario de "Tecnología e Informática" del máster y, con el objeto de avalar la consistencia interna de los resultados también fueron desarrollados por una investigadora externa. En la reunión en la que se definió la codificación inicial se ha contado con un experto externo para recibir asesoramiento y consensuar las subcategorías de análisis desde la mejor adecuación posible (Fleet y Cambourne, 1984). Con todo, posteriormente en el transcurso del proceso de análisis los pares de investigadoras tuvieron que redefinir, en algunos casos, la codificación preliminar ante la emergencia de diferentes subcategorías. Ultimado el análisis por ambas investigadoras se constató que los resultados obtenidos son semejantes.

La fiabilidad de los datos está asociada a la sistematización seguida en el desarrollo del proceso, y particularmente a la del análisis de la información. Atendiendo a Flores (2003) se contemplaron cuidadosamente todos los elementos del diseño metodológico. Asimismo, se presentan evidencias de los resultados con la estructuración oportuna en distintos subepígrafes y tablas, acompañadas de su descripción.

\section{Resultados}

En este apartado exponemos los resultados, desde la perspectiva del alumnado, sobre los beneficios y controversias que presenta la inclusión de las TIC en los centros educativos, así como los cambios que asocian al uso de las mismas. También, incorporamos diferentes fragmentos ilustrativos extraídos de las entrevistas para ofrecer algunos referentes al lector sobre los datos. Se indica para cada fragmento el número de entrevistado, sexo e itinerario del que procede (ejemplo: $\mathrm{E}_{6}$, varón, itinerario de Geografía e Historia).

\section{Beneficios propiciados por las TIC}

Comenzando por el interés que suscita la integración de las TIC en la educación secundaria los entrevistados identifican diferentes beneficios (Tabla 1). Algunas ventajas las asocian con el proceso de enseñanza (para la visualización de contenidos, comunicación bidireccional, disponibilidad de recursos didácticos, enseñanza online, atención a la diversidad, publicación de contenidos y colaboración a través de la Web 2.0); y otras con el de aprendizaje (como el acceso a la información, las posibilidades para la participación y la comunicación online, la generación y/o potenciación de la motivación en el alumnado).

\section{Contribución de las TIC a la enseñanza}

Entre las principales aportaciones de utilizar las TIC en el proceso de enseñanza los participantes se refieren a aquellas que favorecen la visualización de contenidos a través de las presentaciones multimedia y los programas de simulación. La percepción que tienen los entrevistados sobre el uso de las TIC les lleva a sostener que facilitan una mayor claridad y precisión al docente en la exposición oral. Además, argumentan 
que existen innumerables recursos digitales que contribuyen a mejorar el proceso de enseñanza, en particular destacan los beneficios derivados de las aplicaciones de la Web 2.0.

\begin{tabular}{|c|c|c|}
\hline \multicolumn{3}{|c|}{ Subcategoría (de $2^{\circ}$ nivel): Contribución de las TIC al proceso de enseñanza } \\
\hline $\begin{array}{l}\text { Subcategoría } \\
\text { (de } 3^{\circ} \text { nivel) }\end{array}$ & $f$ & Fragmentos ilustrativos \\
\hline $\begin{array}{l}\text { Disponibilidad } \\
\text { de recursos }\end{array}$ & 6 & $\begin{array}{l}\text { «Las TIC son un medio para localizar miles de } \\
\text { recursos didácticos de forma rápida y además } \\
\text { garantizan el éxito.» ( } \mathrm{E}_{6} \text {, varón, itinerario de } \\
\text { Geografía e Historia). }\end{array}$ \\
\hline $\begin{array}{c}\text { Atención } \\
\text { a la diversidad }\end{array}$ & 2 & $\begin{array}{l}\text { «La nuevas tecnologías permiten llegar con más } \\
\text { facilidad al alumnado que tenga algún tipo de } \\
\text { discapacidad. Hoy en día existen muchos } \\
\text { adelantos tecnológicos para darle clase.» ( } \mathrm{E}_{5} \text {, } \\
\text { mujer, itinerario de Tecnología e Informática). }\end{array}$ \\
\hline Enseñanza online & 3 & $\begin{array}{l}\text { «(..) otro recurso interesante es la utilización } \\
\text { de plataformas de elearning en las que los } \\
\text { profesores colocan los temas y las actividades, } \\
\text { así como documentos de consulta y artículos de } \\
\text { revistas o periódicos relacionados.» ( } \mathrm{E}_{9} \text {, mujer, } \\
\text { itinerario de Tecnología e Informática). }\end{array}$ \\
\hline $\begin{array}{c}\text { Publicación de } \\
\text { contenidos, y } \\
\text { colaboración } \\
\text { a través de la Web } 2.0\end{array}$ & 2 & $\begin{array}{l}\text { «El profesor puede estar mejorando } \\
\text { continuamente todos los aspectos de su } \\
\text { actividad profesional, relacionarse y colaborar } \\
\text { con los demás. Además de compartir opiniones } \\
\text { con otros compañeros de su zona, de otros } \\
\text { lugares del país y de otros países. Sin las TIC } \\
\text { sería muy complicado o imposible la } \\
\text { comunicación bidireccional y mediata a } \\
\text { distancia, la colaboración y el intercambio } \\
\text { desinteresado.» (E, varón, itinerario de } \\
\text { Tecnología e Informática). }\end{array}$ \\
\hline $\begin{array}{l}\text { Visualización } \\
\text { de contenidos }\end{array}$ & 12 & $\begin{array}{l}\text { «La utilización de las TIC facilita la labor de } \\
\text { enseñanza al profesor porque le ayuda a } \\
\text { mejorar en la presentación y explicación de los } \\
\text { contenidos empleando gráficos, imágenes, } \\
\text { diagramas, documentos multimedia, etc.» ( } \mathrm{E}_{5} \text {, } \\
\text { varón, itinerario de Geografía e Historia). }\end{array}$ \\
\hline Comunicación & 6 & $\begin{array}{l}\text { «La enorme cantidad de información y riqueza } \\
\text { que facilitan las TIC y sus posibilidades como } \\
\text { medio de comunicación supone una gran } \\
\text { ventaja.» ( } E_{10} \text {, varón, itinerario de Geografía e } \\
\text { Historia). }\end{array}$ \\
\hline
\end{tabular}

Tabla 1. Beneficios asociados al uso de las TIC en la enseñanza 
Los implicados declaran que la utilización de las herramientas digitales facilita y promueve la comunicación online entablada entre los docentes y con el alumnado o entre estos últimos. Consideran que la impartición de la clase se encuentra menos limitada, temporal y espacialmente, porque el profesorado a través de los nuevos recursos tecnológicos puede dejar a disposición de los estudiantes una abundante selección de contenidos, dar respuesta a las dudas que presentan, así como orientarles de forma rápida, personalizada y económica.

Tanto para la enseñanza presencial como en la online los entrevistados valoran positivamente el uso de las herramientas digitales. Por ejemplo, hacen referencia al interés de las plataformas de teleformación por los enormes beneficios que éstas presentan, en particular atendiendo a la reducción de barreras temporales y espaciales. Además sostienen que en la medida en que las instituciones educativas y los docentes habiliten las condiciones oportunas el alumnado podrá utilizar, en mayor medida, los entornos virtuales de aprendizaje en las respectivas asignaturas.

Sobre la publicación de contenidos digitales y la colaboración a través de las aplicaciones de la Web 2.0 los participantes resaltan la importancia de estas herramientas, estimando su potencial para el desarrollo de la interacción por la comunidad educativa y, en general, para la generación de nuevo conocimiento. Con todo, inciden en que además de consumir información es necesario contribuir a su producción, así como a intercambiar recursos y publicar diferentes contenidos para colaborar y crear comunidades online interesantes para el aprendizaje colaborativo.

En relación con la atención a la diversidad, los implicados en el estudio, estiman que las TIC pueden resultar muy útiles para auxiliar al profesorado en la tarea de orientar o guiar al alumnado con algún tipo de discapacidad o problemática puntual, así como para facilitarle información a la familia.

\section{Aportación de las TIC al aprendizaje}

En cuanto a los beneficios sobre la utilización de las TIC que los participantes relacionan con el proceso de aprendizaje del alumnado, como adelantamos, se centran básicamente en la faceta: informativa, participativa o comunicativa y motivacional (Tabla 2).

\begin{tabular}{|c|c|l|}
\hline \multicolumn{4}{|c|}{ Subcategoría (de $2^{\circ}$ nivel $)$ : Aportación de las TIC al aprendizaje } \\
\hline $\begin{array}{c}\text { Subcategoría } \\
\left(\text { de } 3^{\circ} \text { nivel }\right)\end{array}$ & $f$ & \multicolumn{1}{c|}{ Fragmentos ilustrativos } \\
\hline & $f$ & $\begin{array}{l}\text { «Las posibilidades de Internet para la búsqueda de } \\
\text { información son infinitas. Los alumnos tienen recursos muy } \\
\text { importantes para consulta utilizando las enciclopedias } \\
\text { Información }\end{array}$ \\
$\begin{array}{l}\text { Podtimediamos vídeos, fotografías, redes sociales, etc. } \\
\text { herramienta más, que bien utilizada por los estudiantes } \\
\text { mejorará su proceso de aprendizaje y la adquisición de }\end{array}$ \\
\hline
\end{tabular}




\begin{tabular}{|c|c|c|}
\hline & & $\begin{array}{l}\text { conocimientos.» ( } \mathrm{E}_{6} \text {, varón, itinerario de Tecnología e } \\
\text { Informática). }\end{array}$ \\
\hline $\begin{array}{c}\text { Participación } \\
\text { o/y } \\
\text { comunicación }\end{array}$ & 11 & $\begin{array}{l}\text { «Resulta de gran utilidad en secundaria la multitud de } \\
\text { herramientas de Internet para potenciar la participación y } \\
\text { comunicación a través de las plataformas de enseñanza- } \\
\text { aprendizaje, los blogs, foros de debates, etc.» ( } E_{9} \text {, mujer, } \\
\text { itinerario de Geografía e Historia). }\end{array}$ \\
\hline Motivación & 12 & $\begin{array}{l}\text { «El uso de las TIC es muy motivador para el alumnado de } \\
\text { secundaria porque tiene un gran atractivo para estos } \\
\text { chavales. De hecho los puedes cautivar con un } \\
\text { multimedia.» ( } \mathrm{E}_{8} \text {, mujer, itinerario de Tecnología e } \\
\text { Informática). }\end{array}$ \\
\hline
\end{tabular}

Tabla 2. Utilidad de las TIC en el proceso de aprendizaje

Se considera que la cantidad de información que se encuentra disponible en Internet permite, al alumnado de secundaria, un acceso fácil y generalizado a través de motores de búsqueda (indican Google y Yahoo fundamentalmente). Este hecho, estiman que supone una gran ventaja en la medida en que es un avance gigantesco para el proceso de democratización y globalización del conocimiento. Además, argumentan que utilizando los atributos de los recursos digitales los estudiantes interiorizan con mayor facilidad y rapidez determinados contenidos, en particular los de tipo teórico.

Los implicados en el estudio piensan que la participación en el aprendizaje, de los estudiantes de secundaria, se acrecienta con el uso de las TIC y que se refuerza su grado de motivación. Sobre ello añaden que tanto el desarrollo de la colaboración, como el de la motivación se incrementa en los chavales con estos medios. A la vez, resaltan que muchos de los alumnos utilizan las TIC con total normalidad en su vida cotidiana. Es más, el manejo técnico de diferentes dispositivos piensan que, en bastantes casos, es mejor por parte de los adolescentes que por el propio profesorado de secundaria.

\section{Controversias asociadas al uso de las TIC}

El colectivo involucrado en el estudio argumenta que existen diferentes obstáculos para trabajar con las TIC en la enseñanza secundaria. Las limitaciones que han identificado se agruparon en las siguientes subcategorías: insuficiente formación de los docentes, desmotivación del profesorado, carencia de medios técnicos en los centros educativos, y el mal uso que se hace en algunos casos de las TIC (Tabla 3).

\begin{tabular}{|c|c|l|}
\hline \multicolumn{3}{|c|}{ Categoría principal: Controversias asociadas al uso de las TIC } \\
\hline Subcategoría & $\boldsymbol{f}$ & \multicolumn{1}{c|}{ Fragmentos ilustrativos } \\
\hline $\begin{array}{c}\text { Insuficiente } \\
\text { formación de } \\
\text { los docentes }\end{array}$ & 5 & $\begin{array}{l}\text { «Yo creo que si el profesorado no se siente seguro ante las } \\
\text { herramientas digitales, la clase la dará de forma incómoda y las } \\
\text { posibilidades de que los chavales aprendan o incluso se revelen }\end{array}$ \\
\hline
\end{tabular}




\begin{tabular}{|c|c|l|}
\hline & & $\begin{array}{l}\text { serán mucho mayores.» }\left(\mathrm{E}_{6,} \text {, varón, itinerario de Tecnología e }\right. \\
\text { Informática). }\end{array}$ \\
\hline $\begin{array}{c}\text { Desmotivación } \\
\text { del profesorado }\end{array}$ & 7 & $\begin{array}{l}\text { «Hay que tener en cuenta que el profesorado de la enseñanza } \\
\text { pública está muy desmotivado o acomodado cuando lleva } \\
\text { muchos años ejerciendo, y además de caracterizarse en general } \\
\text { por no ser innovador alguno hace lo posible para que otros } \\
\text { tampoco lo sean.» }\left(\mathrm{E}_{7} \text {, mujer, itinerario de Geografía e Historia). }\right.\end{array}$ \\
\hline $\begin{array}{c}\text { Carencia de } \\
\text { medios técnicos }\end{array}$ & 10 & $\begin{array}{l}\text { «Por lo que se puede ver, en los centros educativos de } \\
\text { secundaria uno de los mayores obstáculos para utilizar las } \\
\text { nuevas tecnologías es la propia administración, ya que no aporta } \\
\text { los recursos suficientes.» (E } E_{10} \text { mujer, itinerario de Tecnología e } \\
\text { Informática). }\end{array}$ \\
\hline Mal uso de las & 4 & $\begin{array}{l}\text { «Un inconveniente importante es el mal uso que puede llegar a } \\
\text { hacerse de las TIC, tanto el docente, como el alumnado. La } \\
\text { utilización incorrecta e inoportuna por parte de los chavales } \\
\text { debe ser corregida y orientada por el profesorado. Además, } \\
\text { cuando el docente hace un uso abusivo y sin un objetivo claro la } \\
\text { utilización de las TIC no tiene sentido.» (E, mujer, itinerario de } \\
\text { Tecnología e Informática). }\end{array}$ \\
\hline
\end{tabular}

Tabla 3. Limitaciones ligadas al uso de las TIC

Según los entrevistados una formación inicial y continua de calidad es el mejor medio para garantizar la inclusión de buenas prácticas en los centros, cuando estiman que en la actualidad deja mucho que desear el competencial mediático del profesorado. Además manifiestan que para la inclusión pertinente de las TIC en el curriculum también es importante el interés de los docentes y la actitud de superación permanente, como ingredientes esenciales para mejorar la práctica pedagógica. A su vez, inciden en que los profesionales de la educación han de encontrarse reforzados por la presencia de los recursos digitales en los centros, e impulsar el uso normalizado de los mismos.

En lo relativo al bajo nivel de motivación y dinamismo que atribuyen los participantes a algunos docentes ponen de manifiesto su disconformidad. Además, sostienen que la formación y el trabajo que realizan los educadores ha de estar en continua evolución para integrar estrategias y prácticas creativas e innovadoras con el alumnado de secundaria.

A partir de los comentarios vertidos, los entrevistados argumentan que la mala utilización o la aplicación inadecuada de las TIC, que realizan distintos profesores, desvirtúa las potencialidades con las que cuentan estos medios. Asimismo, la inclusión de los recursos digitales a través de viejas estrategias de enseñanza-aprendizaje estiman que debe evitarse, así como un uso abusivo de los anteriores. Entienden que sin el soporte metodológico apropiado estas herramientas pueden producir la distracción del alumnado y no contribuir suficientemente a su formación. 


\section{Cambios atribuidos al uso de las TIC}

Los participantes identifican diferentes mejoras o cambios asociados a la inclusión de las TIC en secundaria que relacionan con la: diversificación de los recursos digitales, desarrollo de la responsabilidad del alumnado y logros alcanzados en el proceso de enseñanza-aprendizaje (Tabla 4).

\begin{tabular}{|c|c|c|}
\hline \multicolumn{3}{|c|}{ Categoría principal: Cambios atribuidos al uso de las TIC } \\
\hline Subcategoría & $f$ & Fragmentos ilustrativos \\
\hline $\begin{array}{l}\text { Diversificación de } \\
\text { recursos educativos }\end{array}$ & 6 & $\begin{array}{l}\text { «(..) los profesores que utilizan diferentes } \\
\text { recursos y herramientas digitales para impartir } \\
\text { las clases las hacen más interesantes y } \\
\text { productivas para los estudiantes.» ( } \mathrm{E}_{4} \text {, varón, } \\
\text { itinerario de Tecnología e Informática). }\end{array}$ \\
\hline $\begin{array}{l}\text { Desarrollo de la } \\
\text { responsabilidad del } \\
\text { alumnado }\end{array}$ & 4 & $\begin{array}{l}\text { «La integración de las TIC acerca al alumnado a } \\
\text { la vida real y también contribuye a fomentar su } \\
\text { fantasía y socialización. El uso de los recursos } \\
\text { digitales dentro y fuera del aula también supone } \\
\text { una oportunidad para que desarrollen en mayor } \\
\text { medida su responsabilidad, tanto con el cuidado } \\
\text { de los mismos como con su custodia.» ( } \mathrm{E}_{6} \text {, } \\
\text { varón, itinerario de Geografía e Historia). }\end{array}$ \\
\hline $\begin{array}{c}\text { Mejora del proceso } \\
\text { de enseñanza - } \\
\text { aprendizaje }\end{array}$ & 6 & $\begin{array}{l}\text { «(...) algunos recursos tradicionales y las TIC } \\
\text { son la combinación perfecta para mejorar la } \\
\text { formación en secundaria. Además, el uso de las } \\
\text { TIC dinamiza el proceso de enseñanza y } \\
\text { posibilita, en mayor medida, la actualización del } \\
\text { profesorado y la implicación del alumnado y los } \\
\text { docentes.» ( } \mathrm{E}_{2} \text {, mujer, itinerario de Geografía e } \\
\text { Historia). }\end{array}$ \\
\hline
\end{tabular}

Tabla 4. Cambios asociados a las TIC

Los participantes indican que la diversificación de los recursos digitales supone un pilar importante para originar cambios y mejoras en el proceso formativo. Además, el uso de las TIC por el alumnado de secundaria se considera que es una buena forma para desarrollar la corresponsabilidad, con el objeto de evitar o agravar problemas de conducta alimentados por la dependencia o mala utilización de los medios digitales. Asimismo, argumentan que la utilización de los nuevos recursos promueve el trabajo autónomo del alumnado en el ámbito de la competencia de "aprender a aprender", el interés por indagar y por saber más, así como por compartir experiencias. Los implicados sostienen también que la integración de estos recursos en el curriculum de secundaria, entre otros aspectos, permite aprender con facilidad y de forma motivada a los estudiantes.

En general los entrevistados estiman que la inclusión efectiva de las TIC en secundaria produce algunos cambios en las estrategias didácticas y en el tipo de 
actividades desarrolladas. Considerando que aunque no se utilice una metodología innovadora la práctica apoyada por estas herramientas incrementa el interés y la motivación del alumnado por el aprendizaje. Además, piensan que el uso de estos dispositivos genera en el colectivo docente un mayor compromiso, que debe reforzarse con la formación continua, para originar innovaciones auténticas.

\section{Conclusiones y discusión}

Entre los beneficios sobre el uso de los recursos digitales en el proceso de enseñanza los participantes señalan mayores facilidades para la: presentación de contenidos en formato digital, desarrollo de la comunicación bidireccional, disponibilidad y diversidad de medios didácticos, apoyo en la inclusión del alumnado atendiendo a sus diferencias individuales, publicación de contenidos y colaboración a través de herramientas de la Web 2.0. De hecho, las TIC se pueden utilizar para ayudar al profesorado a seguir, regular y controlar los progresos y las dificultades de los estudiantes, así como para apoyar el proceso de retroalimentación y orientar su aprendizaje (Coll, Mauri y Onrubia, 2008). Por otra parte Brown (2010) va más allá al sostener que la Web 2.0 dispone de potencial suficiente para transformar las estrategias didácticas de los docentes.

Asociada a la perspectiva del aprendizaje perciben entre los puntos fuertes de la integración de las TIC en la educación secundaria: facilidades para el acceso a multitud de información, desarrollo de la motivación y posibilidades para la participación y comunicación online. Cuestiones que además de mejorar el nivel de implicación e interacción entre el alumnado y con el profesorado, previsiblemente, contribuirán a la optimización de los resultados académicos (Durán, 2011). La interacción propiciada por las TIC entre los estudiantes es ya una realidad que además contribuye a transformar el rol del profesorado. Resulta inevitable, y también muy oportuno, que los docentes vayan abandonando progresivamente su papel de transmisores de información sustituyéndolo por el de guías y mediadores del aprendizaje. No olvidemos que potenciar los modelos de formación online y crear nuevas propuestas de desarrollo académico es de vital importancia (Suriá, 2010).

Sobre las limitaciones que rodean la integración de los medios digitales en la educación secundaria, a partir de la percepción de los participantes, son de referir: el bajo nivel de motivación de algunos docentes, su insuficiente o deficiente formación técnica y pedagógica, la carencia de medios técnicos en los centros educativos y el mal uso de estos dispositivos. De modo que en algunas instituciones es comprensible la ausencia de utilización de las TIC, ante la deficiente formación que presenta el profesorado y la escasez o falta de actualización de los equipos informáticos.

Cheung (2009) ha apuntado como obstáculos para el uso de los recursos digitales la precariedad de los equipamientos tecnológicos existentes en muchos centros educativos. Además, es necesaria la existencia de una formación más especializada para que los docentes puedan utilizar las TIC y rentabilizar su uso (Ballesta y Lozano, 2007). Por otra parte, Paredes (2009) incide en la importancia de ubicar los recursos 
tecnológicos en espacios compartidos para que pueda beneficiarse de su polivalencia la comunidad educativa.

Como cambios generados por la utilización de las TIC se aprecia la diversificación del uso de los recursos, la generación de un mayor compromiso profesional y el desarrollo de la corresponsabilidad del alumnado. En todo ello los participantes encuentran posibilidades para que mejore el proceso de enseñanza-aprendizaje. En un sentido análogo,

De Pablos, Colas y González (2010) concluyen que la aplicación innovadora de las herramientas digitales facilita en los centros educativos la actitud positiva de los docentes, del equipo directivo y de la comunidad educativa en general, así como la disponibilidad de recursos informáticos o una mayor concienciación y compromiso de los profesionales. En otra investigación se descubren asociados al uso de las TIC cambios superficiales a nivel organizativo, tanto de centro como de aula, sin que sea habitual que conlleven innovación pedagógica en las prácticas docentes (Area, 2010).

\section{Limitaciones y prospectiva}

Sobre las limitaciones de este trabajo y su posible aportación a la comunidad científica ya hemos señalado que por el tipo de estudio presentado no se pretende la generalización de conclusiones, con todo son susceptibles de transferir bajo una mirada crítica (Brown y Yule, 1998; Duarte, 2008). Ahondando en lo que piensa un grupo de participantes, el interés de esta contribución está en el análisis en profundidad que se realiza a partir de los mismos, con el objeto de descubrir su percepción (Bogdan y Biklen, 1982).

Con este trabajo se proporciona, desde otra perspectiva, una visión sobre los pros y los contras de la inclusión de las TIC en la educación secundaria que evidencia una forma de interpretar la realidad. Además, del estudio se desprende que en general las diferentes contribuciones de las TIC se relacionan con dinámicas de cambio y mejora, así como la inclinación revelada por el grupo objeto de análisis hacia un modo de hacer ante la utilización de las herramientas digitales. Lógicamente el presente artículo no agota el tópico abordado sobre el que también estamos desarrollando otros documentos. Del mismo modo, por su relevancia y amplitud animamos a otros investigadores y profesionales a continuar indagando en esta temática.

\section{Referencias bibliográficas}

ALMERICH, G., SUÁREZ, J. M., BELLOCH, C. Y ORELLANA, N. (2010). Perfiles del profesorado a partir del conocimiento de los recursos tecnológicos y su relación con el uso que hacen de estas tecnologías. Revista Complutense de Educación, 21 (2), 247-269. 
AREA, M. (2010). El proceso de integración y uso pedagógico de las TIC en los centros educativos. Un estudio de casos. Revista de Educación, 352, 77-97. http://www.revistaeducacion.mec.es/re352/re352_04.pdf

AZCÁRATE, P. Y CUESTA, J. (2005). El profesorado novel de secundaria y su práctica. Estudio de un caso en las áreas de ciencias. Enseñanza de las Ciencias, 23 (3), 393-402. http://ddd.uab.cat/pub/edlc/02124521v23n3p393.pdf

BALLESTA, F. J. Y LOZANO, J. (2007). Los medios de comunicación ¿nos igualan o nos diferencian? Enseñanza \& Teaching, 25, 45-67.

BOGDAN, R. C. Y BIKLEN, S. K. (1982). Qualitative research for education: an introduction to theory and method. Boston: Allyn and Bacon.

BROWN, G. Y YULE, G. (1998). Discourse analysis. Cambridge: University Press.

BROWN, S. (2010). From VLEs to learning webs: the implications of Web 2.0 for learning and teaching. Interactive Learning Environments, 18 (1), 1-10. http://www.tandfonline.com/doi/pdf/10.1080/10494820802158983

CABERO, J. (1994). Nuevas tecnologías, comunicación y educación. Comunicar, 3, 14-25.

CABERO, J. Y MARTÍN, V. (2011). Campus virtuales compartidos (CVC). Análisis de una experiencia. Educación XX1, 14 (2), 11-132.

CARDONA, J. (2008). La práctica reflexiva, clave en la profesionalización docente. En J. CARDONA (coord.), Cuestiones en torno a la formación y desarrollo profesional de los profesores (183-222). Madrid: Sanz y Torres.

CHEN, H. M., YU, C. Y CHANG, C. S. (2007). E-Homebook System: A web - based interactive education interface. Computers and Education, 49 (2), 160-175. http://www.sciencedirect.com/science/article/pii/S0360131505000886

CHEUNG, C. K. (2009). Media Education Across Four Asian Societies: Issues and Themes. International Review of Education, 55 (1), 39-58. http://www.milthailand.org/phocadownload/media\%20edu\%20across\%204\%20asia $\mathrm{n} \% 20$ societies.pdf

COLL, C., MAURI, T. Y ONRUBIA, J. (2008). Análisis de los usos reales de las TIC en contextos educativos formales: una aproximación sociocultural. Revista Electrónica de Investigación Educativa, $10 \quad$ (1), 1-18. $\mathrm{http} / / /$ redie.uabc.mx/contenido/vol10no1/contenido-coll2.pdf

COLlER, X. (2005). Estudio de casos. Madrid: Centro de Investigaciones Sociológicas.

DE OLIVEIRA, A. T. Y DE LA ROCQUE, G. (2011). O potencial das actividades centradas em produções de alunos na formação de professores de matemáticas. Revista Latinoamericana de Investigación en Matemática Educativa, 14 (3), 335359. 
DE PABLOS, J., COLAS, P. Y GONZÁLEZ, T. (2010). Factores facilitadores de la innovación con TIC en los centros escolares. Un análisis comparativo entre diferentes políticas educativas autonómicas. Revista de Educación, 352, 23-51. $\mathrm{http}: / /$ www.revistaeducacion.mec.es/re352/re352_02.pdf

DUARTE, J. B. (2008). Estudos de caso em Educação. Investigação em profundidade com recursos reduzidos e outro modo de generalização. Revista Lusófona de Educação, 11, 113-132.

DURÁN, J. F. (2011). La contribución del Edublog como estrategia didáctica. Electronic Journal of Research in Educational Psychology, 9 (1), 331-356. http://www.investigacionpsicopedagogica.org/revista/articulos/23/espannol/Art_23_494.pdf

ERTMER, P. A. (2005). Teacher pedagogical beliefs: The final frontier in our quest for technology integration? Educational Technology Research and Development, 53 (4), 25-39. http://blog.lib.umn.edu/cehd/teri/ertmer_2005.pdf

FERNÁNDEZ MARCH, A. (2008). La gestión de la formación del profesorado en la universidad. Teoría de la Educación. Revista Interuniversitaria, 20, 275-312.

FLEET, A. Y CAMBOURNE, B. (1984). The coding of naturalistic data. Research in Education, 41, 1-15.

FLICHY, P. (1995). L'innovation Technique: récents développements en sciences sociales vers une nouvelle théorie de l'innovation. Paris: La Découverte.

FLICK, U. (2004). Introducción a la investigación cualitativa. Madrid: Morata.

FLORES, M. A. (2003). Investigar (com) os professores: reflexões sobre uma pesquisa longitudinal. Perspectiva, 21 (2), 391-412.

FOMBONA; J. Y PASCUAL, M. A. (2011). Las tecnologías de la información y comunicación en la docencia universitaria. Estudio de casos en la Universidad Autónoma de México. Educación XX1, 14 (2), 79-110.

GARCÍA RUÍZ, M. J. (2012). La universidad postmoderna y la nueva creación del conocimiento. Educación XX1, 15 (1), 179-193.

GONZÁLEZ CUBILLÁN, L. (2009). Estudio de casos bajo el enfoque transdisciplinar. Multiciencias, 9 (3), 303-312.

GÜRTLER, L. Y HUBER, G. L. (2007). Should we generalize? Anyway, we do it all the time in everyday life. En L. GÜRTLER, K. MECHTHILD Y G. L. HUBER (eds.), Generalization in Qualitative Psychology (17-35). Tübingen: Verlag Ingeborg Huber.

JAMIESON, P., FISHER, K., GILDING, T., TAYLOR, P. Y TREVITT, A. C. F. (2000). Place and space in the design of new learning environments. Higher Education Research and Development, 19 (2), 221-237. 
LATORRE, A. (2007). La investigación-acción. Conocer y cambiar la práctica educativa. Barcelona: Grao.

LITTLE, J. W., GEARHART, M., CURRY, M. Y KAFKA, J. (2003). Looking at student work for teacher learning, teacher community, and school reform. Phi Delta Kappan, 85 (3), 184-192.

LÓPEZ RUÍZ, J. I. (2011). Un giro copernicano en la enseñanza universitaria: formación por competencias. Revista de Educación, 356, 279-301.

MARCELO, C. (2009). Formalidad e informalidad en el proceso de aprender a enseñar. Revista de Educación, 350, 31-55. http://www.revistaeducacion.mec.es/re350/re350_02.pdf

MCMILLANM, J. Y SHUMACHER, S. (2005). Investigación educativa una introducción conceptual. Madrid: Pearson.

MILES, M. M. Y HUBERMAN, A. M. (1984). Qualitative data analysis: A sourcebook of new methods. Newbury Park: Sage.

MURGA, M. A. Y GARCÍA, I. (2010). La carta de la tierra y el blog: dos elementos centrales de una experiencia docente innovadora en $4^{\circ}$ de ESO. Enseñanza de las Ciencias, 28 (1), 85-94. http://ensciencias.uab.es/revistes/28-1/085-094.pdf

PAREDES, J. (2009). Materiales para la innovación educativa dentro y fuera del aula. EN J. PAREDES Y A. DE LA HERRÁN (coords.), La práctica de la innovación educativa (311-326). Madrid: Síntesis.

PERRENOUD, F. (2007). Desarrollar la práctica reflexiva en el oficio de enseñar. Barcelona: Grao.

PORLÁN, R., MARTÍN, M. R., RIVERO, A., HARRES, J., AZCÁRATE, P. Y PIZZATO, M. (2010). El cambio del profesorado de ciencias I: marco teórico y formativo. Enseñanza de las Ciencias, 28 (1), 38-42. http://ensciencias.uab.es/revistes/28-1/031-046.pdf

SANDOVAL, E. (2009). La inserción a la docencia. Aprender a ser maestro de secundaria en México. Profesorado. Revista de Curriculum y Formación del Profesorado, 13 (1), 183-194. http://redalyc.uaemex.mx/pdf/567/56711733013.pdf

SIERRA, B. Y PÉREZ FERRA, M. (2007). La comprensión de la relación de la teoría-práctica: una clave epistemológica de la didáctica. Revista de Educación, 342, 553-576. http://www.revistaeducacion.mec.es/re342/re342_25.pdf

STAKE, R. (2007). A arte de investigação com estudos de caso. Lisboa: Fundação Calouste Gulbenkian.

SUÁREZ PAZOS, M. Y MEMBIELA, P. (2011). Nostalgia en la escuela rural en los recuerdos de sus antiguos estudiantes. Profesorado. Revista de Curriculum y Formación del Profesorado, 15 (2), 315-325. http://www.ugr.es/ recfpro/rev152COL8.pdf 
SURIÁ, R. (2010). Las TIC en las titulaciones universitarias de grado: análisis del conocimiento y uso en el alumnado de la Universidad a Distancia. Electronic Journal of Research in Educational Psychology, 8 (3), 1179-1200. http://www.investigacionpsicopedagogica.org/revista/new/ContadorArticulo.php?477

VALE, I. (2000). Didáctica da matemática e formação inicial de professores num contexto de resolução de problemas e de materiais manipuláveis. Aveiro: Universidade de Aveiro.

YIN, R. (2006). Case Study Methods. En J. L. GREEN, G. CAMILI Y P. B. ELMORE (eds.), Handbook of Complementary Methods in Education Research (111-122). Mahwah: Erlbaum.

\section{Correspondencia con las autoras}

$\mathrm{M}^{\mathrm{a}}$ Carmen Ricoy Lorenzo y Maria João V. S. Couto

Facultad de C.C. de la Educación, Universidad de Vigo

Avd. Castelao, s/n, C.P. 32004, Ourense (España)

Teléfono: 988387065

E-mail: cricoy@uvigo.es; mjoaocouto@uvigo.es 